\title{
Intraventricular Neurocysticercosis And Bruns Syndrome: A Case Report
}

\author{
Banskota NP ${ }^{1}$, Singh $\mathrm{K}^{2}$
}

\begin{abstract}
Neurocysticercois is a common neurological infection but intraventricular NCC is rare even in endemic areas. Here we discuss a case of intraventricular NCC located at foramen of monro leading to Bruns syndrome.
\end{abstract}

\section{INTRODUCTION}

Cysticercosis refers to infection by the larval stage of the pork tapeworm Taenia solium. It clinically manifests as neurocysticercosis (NCC) and extraneural cysticercosis. NCC is divided into parenchymal and extraparenchymal forms; the latter includes intraventricular, subarachnoid, and occasionally spinal. Intraventricular NCC (IVNCC) can be rapidly progressive and fatal if untreated. NCC is the most common helminthic neurological infection in the world, and one of the most frequent causes of adultonset epilepsy and hydrocephalus worldwide Globally, NCC is reported to affect approximately 50 million individuals, mainly in Latin America, sub-Saharan Africa, and South and Southeast Asia ${ }^{2}$. Brain parenchymal involvement occurs in $60 \%$ to $92 \%$ of patients with NCC, but intraventricular lesions are seen in only 7 to $20 \%$ of cases, out of which the lesions are more commonly seen in the $4^{\text {th }}$ ventricle (54-64\%), followed by the $3^{\text {rd }}$ ventricle (23-27\%), the lateral ventricles (11-14\%) and Sylvian aqueduct (9\%) ${ }^{7,89}$.

Bruns syndrome was first described in 1902, as a sudden onset of severe headaches and vomiting associated to a vestibular syndrome provoked by abrupt change in head position. It is related to an episodic obstructive hydrocephalous caused by an intraventricular mass that acts like a ball-valve mechanism ${ }^{3}$.

\section{CASE REPORT}

A 10 years old girl was presented with complaints of severe intractable headache, vomiting, blurring of vision, altered sensorium and seizure. On examination she had strabismus. CT head was ordered which showed gross hydrocephalus and an hyper dense small round mass was seen at junction of lateral ventricles [Figure 1]. She was prepared for surgery, Ventriculo peritoneal shunting was performed. Post-operative period was uneventful. Her headache, visual disturbances were back to normal. CT showed normal size ventricles and cyst dislodged from its original position. [Figure 3].CSF report was normal. After a week she was discharged. Counseling was done about the care of shunt.

1. Dr. Narendra Prasad Baskota

2. Dr. K. Singh

Address for correspondence: Dr. Narendra Prasad Baskota Consultant Department of Neurosurgery Nepalgunj Medical College \& Teaching Hospital Kohalpur, Banke, Nepal

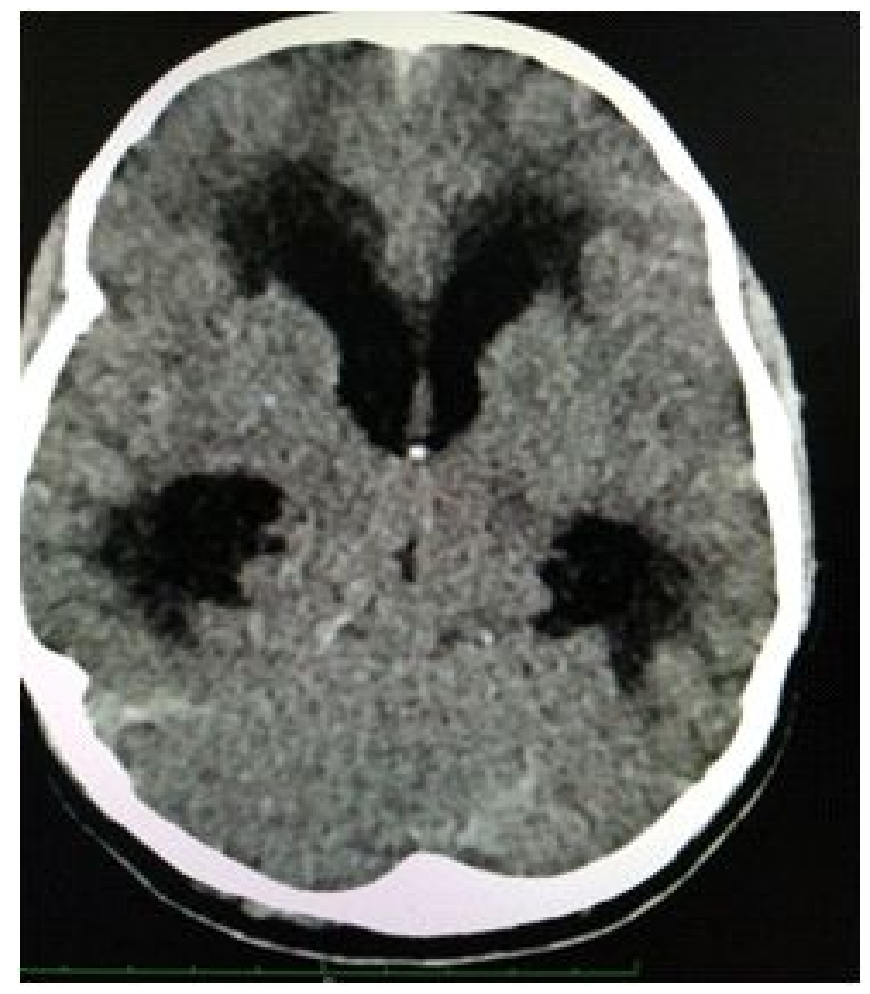

Figure 1: CT head showing dilated lateral ventricles with hyperdense mass(NCC) at foramen of monro 


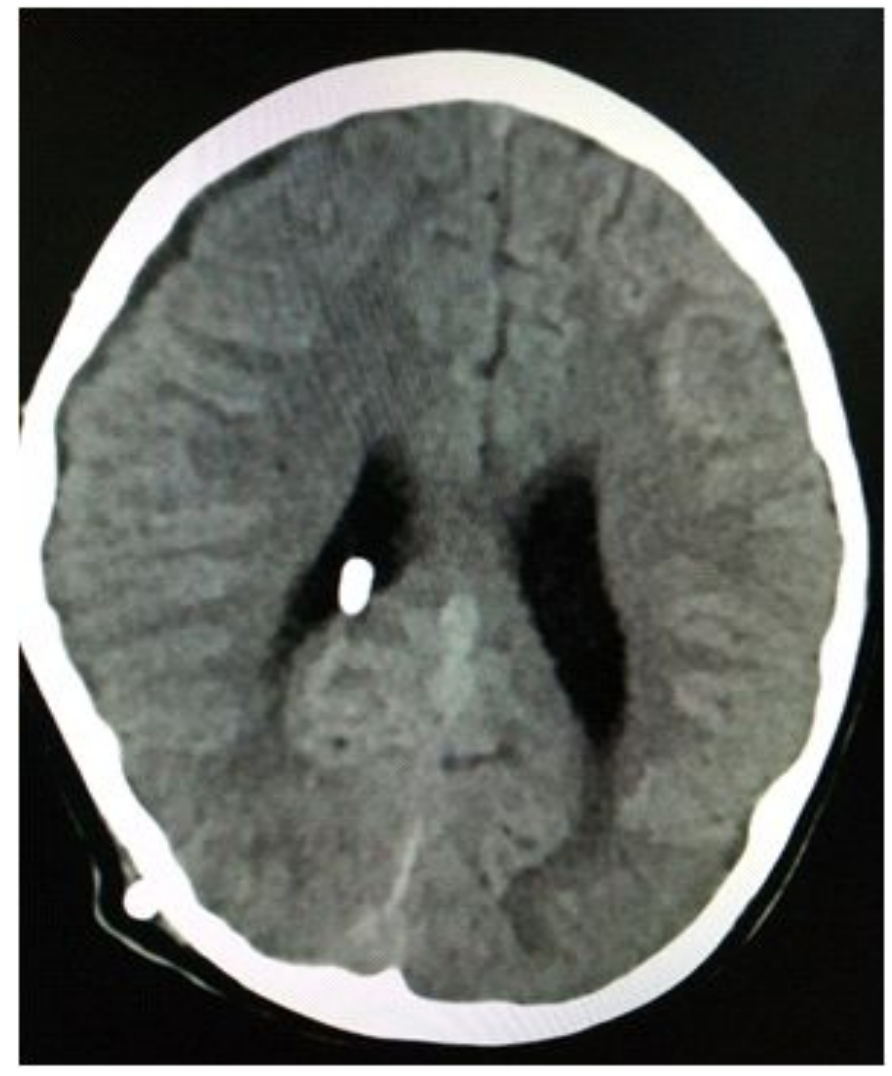

Figure 2: CT head done after placement of VP shunt

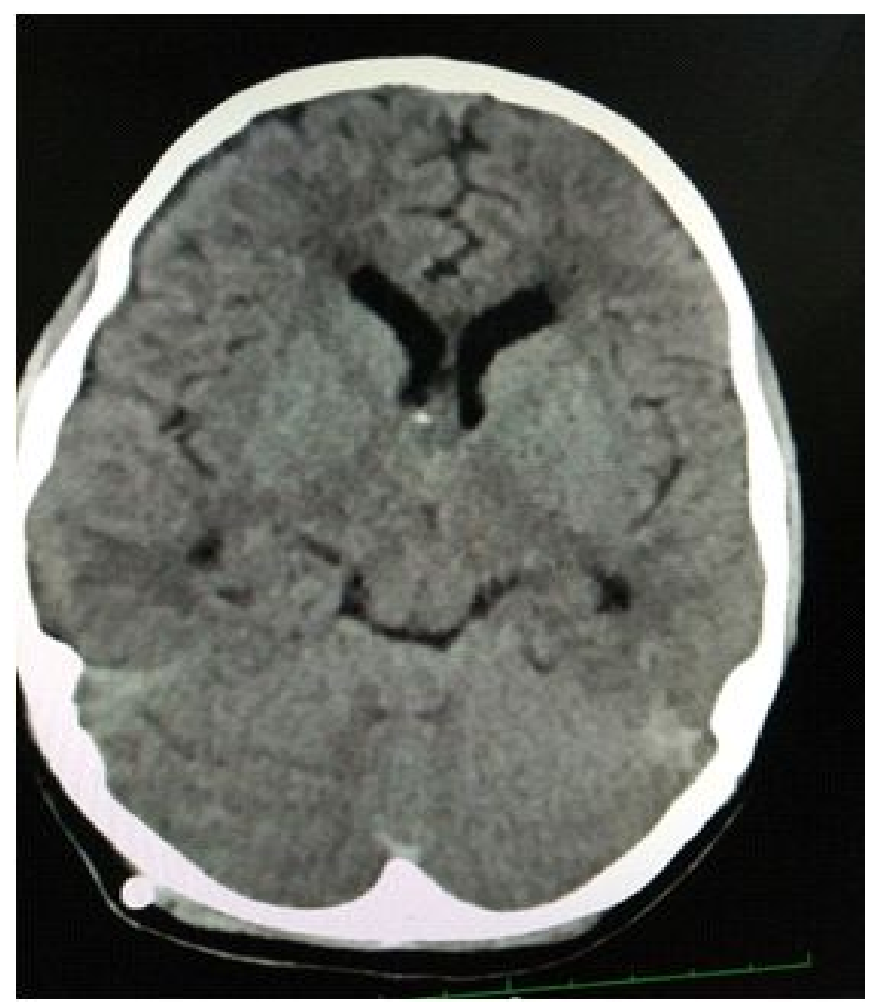

Figure 3: Post operative CT showing normal sized ventricles and cyst displaced

\section{DISCUSSION}

Neurocysticercosis is a very common neurological infection. The cysticercus larva (after embedding itself in the parenchyma) undergoes four stages of evolution: Vesicular, colloidal, granulo-nodular, and nodular- calcified. This evolution does not occur in the intraventricular and the subarachnoid form of NCC ${ }^{1}$.IVNCC could present with various features; however, life-threatening obstructive hydrocephalus caused by positional CSF obstruction (Bruns syndrome) is an infrequent and striking feature of the disease

The Bruns syndrome is an unusual phenomenon, characterized by attacks of sudden and severe headache, vomiting, and vertigo, triggered by abrupt movement of the head. The presumptive cause of Bruns syndrome is a mobile deformable intraventricular mass leading to an episodic obstructive hydrocephalus resulting from an intermittent or positional cerebral spinal fluid (CSF) obstruction with elevation of intracranial pressure due to a ball valve mechanism ${ }^{5}$. Intraventricular neurocysticercosis (IVNCC) has been reported as an important etiology of Bruns syndrome4.

In this case also the patient had IVNCC and presented with attacks of severe intractable headache, vomiting, vertigo triggered by head movement. In such patients medical management is not the choice of treatment. Decreasing the CSF pressure is very important. Our patient also underwent surgery, ventriculo peritoneal shunting was done. After that symptoms were subsided. Endoscopic neurosurgery is not available in our institution so removal of cyst could not be done. Patient was asymptomatic after 2 months follow up.

\section{CONCLUSION}

Although neurocysticercosis is a common neurological infection, intraventricular NCC is very rare. We found a case of IVNCC with Bruns syndrome and treated with VP shunt.

\section{REFERENCES}

1. Intraventricular racemose type neurocysticercosis with anterior interhemispheric fissure cyst:A case report. J Neurosci Rural Pract. 2015 Apr-Jun;6(2):234-237

2. World Health Organization. Working to overcome the global impact of Neglected Tropical Diseases. First Report on Neglected Tropical Diseases. Geneva, Switzerland: World Health Organization 2010. Available: http://www.who.int/neglected_diseases/2010report/en/ 
. Accessed 29 October 2016.

3. Bruns syndrome:a deadly sign. Pan Afr Med J. 2015;22:229

4. Diehl Rodriquez R, Crestani DN, Dworzecki Soares JO, Franceshini PR, Petersen Alves R, Zimerman R, Ferreira N, Menke Barea L. Bruns' syndrome and racemose neurocysticercosis: a case report. Rev Soc Bras Med Trop. 2012;45:269-271.

5. Bruns L. Neuropathologische demonstrationen. Neurol Centralbl. 1902;21:561-567.

6. Intraventricular Taenia solium Cysts Presenting with Bruns Syndrome and Indications for Emergent Neurosurgery Lokesh Shahani, Natalie Dailey Garnes, and Rojelio Mejia“Am J Trop Med sHyg. 2015 Jun 3; 92(6): 1261-1264.

7. . Kalra S, Jaiswal AK, Behari S, Jain VK. Lateral ventricular neurocysticercosis: A case report. Indian J Radiol Imaging. 2006;16:775-8.

8. Citow JS, Johnson JP, McBride DQ, Ammirati M. Imaging features and surgery-related outcomes in intraventricular neurocysticercosis. Neurosurg Focus. 2002;12:6e.

9. Schantz PM, Tsang VC, Maddison SE. Serodiagnosis of neurocysticercosis. Rev Infect Dis. 1998;10:1231-3. 